\title{
ROBUSTNESS OF FLEXIBLE SYSTEMS WITH COMPONENT-LEVEL UNCERTAINTIES
}

\author{
Peiman G. Maghami * \\ NASA Langley Research Center, Hampton, VA 23681
}

\begin{abstract}
Robustness of flexible systems in the presence of model uncertainties at the component level is considered. Specifically, an approach for formulating robustness of flexible systems in the presence of frequency and damping uncertainties at the of flexible systems in the presence The synthesis of the components is based on a modifications of a controls-based algorithm for component mode synthesis. The modifications of a controls-based algors of synthesized flexible systems. It is then extended to deal with global (non-synthesized) dynamic models with component-level uncertainties by projecting uncertainties from component levels to system level. A numerical example involving a two-dimensional simulated docking problem is worked out to demonstrate the feasibility of the proposed approach.
\end{abstract}

\section{Introduction}

Component mode synthesis is a well established area in modeling of flexible systems ${ }^{1-6}$. There are several advantages of modeling via component mode synthesis. These include: (1) It allows much independence in the design and analysis of components, i.e., the work can be performed by different groups or organizations. A good example is the International Space Station which are built by different companies in several countries. (2) It increases the power of existing finite-element analysis and design, by allowing it to handle excessively large problems; (3) It allows a direct synthesis of substructure test data. This is particularly useful for very large structural systems (such as the International Space Station) that cannot be tested as a whole. It can also be used as a part of an experimental verification tool for substructures before deployment as a connected structure, for example, to provide uncertainty models for the component dynamics.

In applications of robust control to flexible systems, uncertainty models have been typically assumed or defined for the combined (full-up) system. This approach is acceptable so long as these uncertainties (in terms of structures and levels) have been verified through experimental work. However, this is not the case in many situations. A good example is where ground testing of the assembled

\footnotetext{
* Senior Research Engineer, Guidance and Control Branch Senior Member AIAA.

Copyright 2000 by the American Institute of Aeransutics and Astronautics. Inc No copyright is agserted in the United States under Title 17 . all rights All other rights are reserved by the copyright owner.
}

structure is not possible, i.e., only component-based testing is feasible. In such cases, reliable uncertainty models can only be defined for the components. Consequently, methods are needed to provide for robust design and analysis of flexible systems with the uncertainty defined at the component levels.

In this paper, robustness of flexible systems with component level uncertainties are addressed. First, robustness of a synthesized system to uncertainties in the frequency and damping of its components is considered. The approach used for component synthesis is based on a slight modification of the control-based synthesis algorithm by Maghami and Lim. ${ }^{7}$ The synthesized plant in transformed coordinates is placed in a Linear Fractional Transformation (LFT) form for robust design and analysis. Next, a formulation is also presented through which the uncertainties at the component level can be extended to system level uncertainties for design or analysis. This is quite useful in situations wherein while a global model of the assembled system is available, uncertainties are defined for the components only. The proposed approach is applied to a twodimensional problem involving the docking of two components in space.

\section{Substructure Synthesis}

Assume that two flexible systems are connected as shown in Fig. 1. These systems may represent two components of a larger flexible body (such as spacecraft or aircraft), or they may represent two self-sufficient systems that have joined for a specific purpose, such as two spacecraft docking. Following the approach of Maghami and $\operatorname{Lim},{ }^{7}$ the interface 


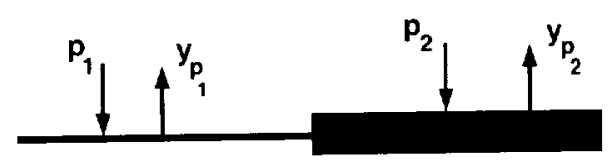

Flexible System

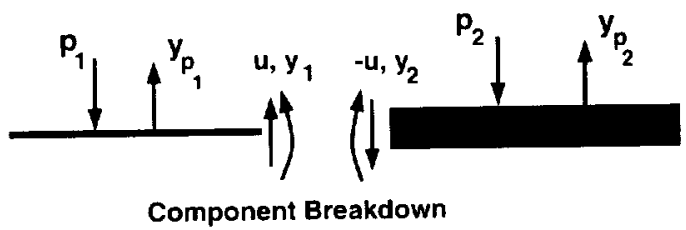

Fig. 1 Diagram of the Two-Component System

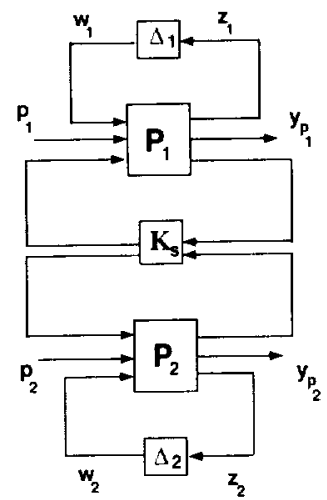

Fig. 2 Block Diagram of the Two-Component System

forces and moments (between the two components) may be considered as control input forces. A block diagram for the system is illustrated in Fig. 2. Here, each of the component plants are represented by $P_{1}$ and $P_{2}$, respectively. Associated with each component model, there is uncertainty in the Linear Fractional Transformation (LFT) form, representing parametric and nonparametric uncertainties in the component models. The controller, $K_{s}$, denotes the controller that synthesizes the combined dynamics of two components in a joined configuration. The state-space representation of the dynamics of the components is

$$
\begin{gathered}
\text { component } 1 \\
\dot{x}_{1}=A_{1} x_{1}+B_{1} u+H_{1} p_{1}+E_{1} w_{1} \\
y_{1}=C_{1} x_{1} \\
z_{1}=F_{1} x_{1} \\
y_{p_{1}}=L_{1} x_{1}+D_{u_{1}} u+D_{p_{1}} p_{1}
\end{gathered}
$$

$$
\begin{gathered}
\text { component } 2 \\
\dot{x}_{2}=A_{2} x_{2}+B_{2} u+H_{2} p_{2}+E_{2} w_{2} \\
y_{2}=C_{2} x_{2} \\
z_{2}=F_{2} x_{2} \\
y_{p_{2}}=L_{2} x_{2}+D_{u_{2}} u+D_{p_{2}} p_{2}
\end{gathered}
$$

where $A_{i}, i=1,2$ denote the state matrices for each of the components. Matrices $B_{i}, H_{i}, E_{i}, C_{i}, F_{i}$, and $L_{i}, i=1,2$, represent the influence matrices for interface inputs, non-interface inputs, uncertainty outputs, interface outputs, uncertainty inputs, and non-interface output for each component, respectively. The matrices $D_{u_{1}}, D_{p_{1}}, D_{u_{2}}$, and $D_{p_{2}}$, represent feedthrough matrices associated with non-interface outputs of each component; $x_{1}$ and $x_{2}$ denote the state vectors of the components; $y_{1}$ and $y_{2}$ denote the interface output vectors, including the displacements and velocities at the interface from each component; $y_{p_{1}}$ and $y_{p_{2}}$ denote the non-interface output vectors components which includes performance outputs as well as measurement outputs for local feedback control; $z_{1}$ and $z_{2}$ denote the uncertainty input vectors; $p_{1}$ and $p_{2}$ represent the non-interface inputs which include exogenous disturbances as well as control inputs for local feedback control; and $w_{1}$ and $w_{2}$ denote the uncertainty output vector. The influence matrices $E_{i}$ and $F_{i}, i=1,2$ corresponding to component uncertainties will be defined later.

Now, the derivation in Ref. ${ }^{7}$ is followed to obtain the synthesized dynamics of the combined system. However, in this paper, the interface output vector includes the interface velocity vector as well as the displacement vector at the interface. The motivation behind including the displacement vectors is to avoid the superfluous rigid-body mode that may come about from the previous formulation. Let $N_{c}$ denote an orthonormal basis for the right null space of $\left[\begin{array}{ll}C_{1} & -C_{2}\end{array}\right]$, i.e.,

$$
\left[\begin{array}{ll}
C_{1} & -C_{2}
\end{array}\right] N_{c}=0
$$

and let $R_{c}$ denote an orthonormal complement to $N_{c}$. Transform the combined system via an orthogonal similarity transformation, such that

$$
\left\{\begin{array}{l}
x_{1} \\
x_{2}
\end{array}\right\}=\left[\begin{array}{ll}
N_{c} & R_{c}
\end{array}\right]\left\{\begin{array}{c}
\alpha \\
\beta
\end{array}\right\}
$$

Then, the dynamics of the combined system in transformed coordinates becomes

$$
\dot{\alpha}=\hat{A}_{1} \alpha+\hat{H}_{1} p+\hat{E}_{1} w
$$




$$
\begin{gathered}
z \equiv\left\{\begin{array}{l}
z_{1} \\
z_{2}
\end{array}\right\}=\hat{F}_{1} \alpha \\
y_{p} \equiv\left\{\begin{array}{l}
y_{p_{1}} \\
y_{p_{2}}
\end{array}\right\}=\hat{N}_{1} \alpha+\hat{D}_{1} p \\
\text { where } p=\left\{\begin{array}{c}
p_{1} \\
p_{2}
\end{array}\right\}, w=\left\{\begin{array}{c}
w_{1} \\
w_{2}
\end{array}\right\} \text {, and } \\
\hat{F}_{1}=\left[\begin{array}{cc}
F_{1} & 0 \\
0 & F_{2}
\end{array}\right] N_{c} \\
\hat{N}_{1}=\left[\begin{array}{cc}
L_{1} & 0 \\
0 & L_{2}
\end{array}\right] N_{c}-\left[\begin{array}{c}
D_{u_{1}} \\
D_{u_{2}}
\end{array}\right] \hat{B}_{2}^{-1} \hat{A}_{3} \\
\hat{D}_{1}=\left[\begin{array}{cc}
D_{p_{1}} & 0 \\
0 & D_{p_{2}}
\end{array}\right]-\left[\begin{array}{l}
D_{u_{1}} \\
D_{u_{2}}
\end{array}\right] \hat{B}_{2}^{-1} \hat{H}_{2}
\end{gathered}
$$

and

$$
\left[\begin{array}{ll}
\hat{A}_{1} & \hat{A}_{2} \\
\hat{A}_{3} & \hat{A}_{4}
\end{array}\right]=\left[\begin{array}{ll}
N_{c} & R_{c}
\end{array}\right]^{T}\left[\begin{array}{cc}
A_{1} & 0 \\
0 & A_{2}
\end{array}\right]\left[\begin{array}{cc}
N_{c} & R_{c}
\end{array}\right]
$$

$$
\begin{gathered}
{\left[\begin{array}{l}
\hat{B}_{1} \\
\hat{B}_{2}
\end{array}\right]=\left[\begin{array}{ll}
N_{c} & R_{c}
\end{array}\right]^{T}\left[\begin{array}{c}
B_{1} \\
B_{2}
\end{array}\right]} \\
{\left[\begin{array}{c}
\hat{H}_{1} \\
\hat{H}_{2}
\end{array}\right]=\left[\begin{array}{ll}
N_{c} & R_{c}
\end{array}\right]^{T}\left[\begin{array}{cc}
H_{1} & 0 \\
0 & H_{2}
\end{array}\right]} \\
{\left[\begin{array}{l}
\hat{E}_{1} \\
\hat{E}_{2}
\end{array}\right]=\left[\begin{array}{ll}
N_{c} & R_{c}
\end{array}\right]^{T}\left[\begin{array}{cc}
E_{1} & 0 \\
0 & E_{2}
\end{array}\right]}
\end{gathered}
$$

\section{Model Uncertainty}

The synthesized dynamics of the combined system is illustrated in Fig. 3. Here, the plant $P$, representing the system dynamics, is given as

$$
\left\{\begin{array}{c}
\dot{\alpha} \\
y_{p} \\
z
\end{array}\right\}=\left[\begin{array}{ccc}
\hat{A}_{1} & \hat{H}_{1} & \hat{E}_{1} \\
\hat{N}_{1} & \hat{D}_{1} & 0 \\
\hat{F}_{1} & 0 & 0
\end{array}\right]\left\{\begin{array}{c}
\alpha \\
p \\
w
\end{array}\right\}
$$

The uncertainty block, $\Delta$ include uncertainties from both components, i.e.,

$$
\Delta=\left[\begin{array}{cc}
\Delta_{1} & 0 \\
0 & \Delta_{2}
\end{array}\right]
$$

The uncertainties represented by $\Delta$ may include parametric and nonparametric uncertainties in each of the components. Parametric uncertainties include uncertainties in modal frequencies, damping ratios, and mode shapes of the components. The nonparametric uncertainties considered here are the unmodeled dynamics of the components, which would typically be due to the truncated modes of the components. In this paper, parametric uncertainties in the component models in the form of frequency and damping uncertainties are considered.

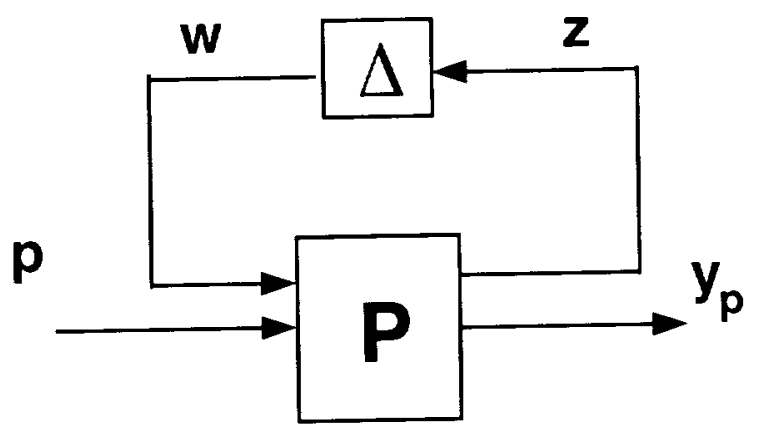

Fig. 3 Block Diagram of the Synthesized System

\section{Uncertainty in Frequency and Damping}

If, as typically done, a modal model of the components are used to describe their dynamics, then uncertainties in modal frequency and damping are embedded in the elements of the component state matrices $A_{1}$ and $A_{2}$. Consider the first component, and assume that there are $n_{1}$ distinct uncertainties associated with the $A_{1}$. Then, one may write

$$
A_{1} \rightarrow A_{1}+\sum_{i=1}^{n_{1}} Q_{i} \delta_{i}
$$

where matrix $Q_{i}$ represents the influence matrix for the $\delta_{i}$ uncertainty. Now, the influence matrices $E_{1}$ and $F_{1}$ (see Eqs. (1) and (3)) may be obtained from decomposition of matrices $Q_{i}, i=1, \ldots, n_{1}$. Factor each matrix as

$$
Q_{i}=E_{1 i} F_{1 i}
$$

where $E_{1 i} \in R^{n \times r_{i}}, E_{1 i} \in R^{r_{i} \times n}$, and $r_{i}$ denotes the rank of $Q_{i}$. Singular Value Decomposition (SVD) is a good way of performing this factorization. Then, the influence matrices $E_{1}$ and $F_{1}$ may be written as

$$
E_{1}=\left[\begin{array}{llll}
E_{11} & E_{12} & \ldots & E_{1 n_{1}}
\end{array}\right]
$$

$$
F_{1}=\left[\begin{array}{c}
F_{11} \\
F_{12} \\
\vdots \\
F_{1 n_{1}}
\end{array}\right]
$$

With matrices $E_{1}$ and $F_{1}$ defined, the synthesized system represented by Eq. (21) is in suitable form for robustness analysis or control design.

\section{Component Uncertainty at System Level}

In some applications, it may be the case that an overall model of the combined structure is available either from full-up structural analysis or system identification, i.e., no component synthesis is required to generate the model. However, the 
model uncertainty is lefined at the component level, where substantially more effort is typically spent for modeling, testing, and verification. In order to perform robustness analysis or robust control design, the uncertainty formulation has to redefined at the system level. Assume, without any loss of generality, that the overall model at the system level is in a real modal form. Then, the model of the system may be written as

$$
\left\{\begin{array}{c}
\dot{x}_{s} \\
y_{p} \\
z
\end{array}\right\}=\left[\begin{array}{ccc}
A_{s} & H_{s} & E_{s} \\
N_{s} & D_{s} & 0 \\
F_{s} & 0 & 0
\end{array}\right]\left\{\begin{array}{c}
x_{s} \\
p \\
w
\end{array}\right\}
$$

Here, $A_{s}$ has the following form

$$
A_{s}=\left[\begin{array}{cccc}
A_{s_{1}} & 0 \ldots & 0 & \\
0 & \ddots & \ldots & 0 \\
\vdots & & \ddots & \vdots \\
0 & \ldots & 0 & A_{s_{m}}
\end{array}\right]
$$

with

$$
A_{s_{i}}=\left[\begin{array}{cc}
\lambda_{i_{R}} & -\lambda_{i_{I}} \\
\lambda_{i_{I}} & \lambda_{i_{R}}
\end{array}\right]
$$

where $\lambda_{i_{R}}$ and $\lambda_{i_{I}}$ denote the real and imaginary part of the ith eigenvalue pair of the system. Here, we assume that the eigenvalues are ordered from smallest to the largest. From component synthesis, we know that the first $m$ eigenvalues of matrix $\hat{A}_{1}$ should closely match the eigenvalues of the $A_{s} . m$ is typically chosen as the number of system modes that are within half the bandwidth of the component dynamics. Let matrix $\chi$ denote a real similarity transformation through which matrix $\hat{A}_{1}$ is put in real modal form, with eigenvalue blocks in increasing order, and let $\Psi=\chi^{-1}$. Such a transformation may be obtained from the complex eigenvectors of $\hat{A}_{1}$ by collecting the real and imaginary parts of the eigenvectors corresponding to complex conjugate pairs of eigenvalues. Applying this similarity transformation to the system in Eq. (21) gives

$$
\left\{\begin{array}{c}
\dot{\gamma} \\
y_{p} \\
z
\end{array}\right\}=\left[\begin{array}{ccc}
\Psi \hat{A}_{1} \chi & \Psi \hat{H}_{1} & \Psi \hat{E}_{1} \\
\hat{N}_{1} \chi & \hat{D}_{1} & 0 \\
\hat{F}_{1 \chi} & 0 & 0
\end{array}\right]\left\{\begin{array}{c}
\gamma \\
p \\
w
\end{array}\right\}
$$

Comparing the system in Eq. (30) with that given by Eq. (27), and keeping in mind that the first $m$ eigenvalues of the two systems should match, it is observed that the uncertainties at the component level can not be put exactly in the same structure at the system level, mainly due to the additional
Component No. 1

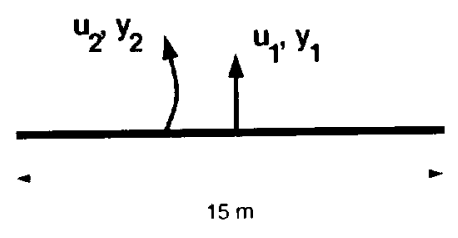

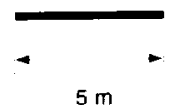

Component No. 2
Fig. 4 Components of the Free-Free Problem

dynamics in the synthesized model that is neglected. However, a reasonable approximation may be made by choosing $E_{s}$ and $F_{s}$ as

$$
E_{s}=\Psi_{1} \hat{E}_{1} ; \quad F_{s}=\hat{F}_{1} \chi_{1}
$$

where $\Psi_{1}$ and $\chi_{1}$ denote partitions formed from the first $2 \times m$ rows and $2 \times m$ columns of $\Psi$ and $\chi$, respectively. With $E_{s}$ and $F_{s}$ defined, the global system is prepared for robust design and analysis. It should be noted that if more than one rigid-body mode are present in the system (e.g., space applications), then it would be unlikely that the a rigid-body mode in $\hat{A}_{1}$ would have a exact corresponding mode in $A_{s}$. This is because the eigenvectors corresponding to rigid-body modes (with repeated eigenvalues at zero) are not unique. One way to handle this is to replace the partition in matrices $A_{s}, H_{s}$, and $N_{s}$, corresponding to the rigid-body modes, with those of $\Psi \hat{A}_{1} \chi, \Psi \hat{H}_{1}$, and $\hat{N}_{1} \chi$, respectively. This note also applies to repeated flexible modes, except in many of such cases unique eigenvectors may exist.

\section{Numerical Results}

The proposed approach for robust design and analysis under component-level uncertainty models is applied to a two-component planar problem shown in Figure 4. Here, a docking scenario involving two flexible space systems is considered. The two systems are modeled as planar free-free Euler-Bernoulli beams. The material and geometric properties of the system were chosen to provide considerable modal content in the low-mid frequency range, to make the synthesis and control design task more challenging, and are provided in Table 1 . It is assumed that in the docked configuration component no. 1 would provide attitude and position control for the combined system. 
Table 1. Geometric and structural properties

\begin{tabular}{cccc} 
Property & Comp. 1 & Comp. 2 & Whole \\
\hline Length & 15 & 5 & 20 \\
Mass/Length & 6.775 & 6.775 & 6.775 \\
Rigidity, EI & 36458.3 & 36458.3 & 36458.3 \\
No. of modes & 20 & 20 & 20 \\
\hline
\end{tabular}

\section{Control Design}

The control design for component no. 1 is briefly described. As shown in Fig. 4, there are two control inputs available for this component. There is a torque wheel at the third of the length $(x=5 \mathrm{~m})$ and there is a thruster at the middle of the beam $(\mathrm{x}=7.5 \mathrm{~m})$ to provide attitude and position control. Collocated with these actuators are attitude and position sensors, providing measurement for the feedback loop. Here, sensors and actuators dynamics are not considered. The control design was based on the $H_{\infty}$ synthesis. The performance requirements were defined in terms of the weighted output sensitivity transfer function from disturbances to the measurement sensors. The weighting function was chosen as $W_{p}=\frac{0.002(s+100)}{(s+0.0001)(s+10)}$ for each channel to provide good disturbance rejection at low to mid frequencies as well as to provide integral action to minimize steady-state errors. Uncertainty in the system model, in the form of unstructured input multiplicative, was used. The weighting function, used to scale or distribute the uncertainty, was chosen as $W_{u}=\frac{(s+50)}{(s+500)}$ to emphasize model uncertainty in the mid-high frequency range. A 10order model of the beam, which included the first three flexible modes, were used in the control design. The controller was synthesized using the "hinfsyn" routine of $\mu$-Analysis and Synthesis Toolbox. ${ }^{8}$ The $H_{\infty}$ design converged at a $\gamma$ value of 0.942 (i.e., all the design requirements were met), and resulted in a 16th-order controller. Figure 5 provides a bode plot of the controller. This controller was later used in robustness analysis of the system in the docked configuration.

\section{Robustness Analysis}

Here, the two components were assumed to be in a docked configuration. Each of the components was modeled using the first 20 modes (including two rigid-body modes and 18 flexible modes), resulting in a 40th-order state space model for each of the substructures. The 20 modes used were deemed sufficient to provide a basis for describing the first
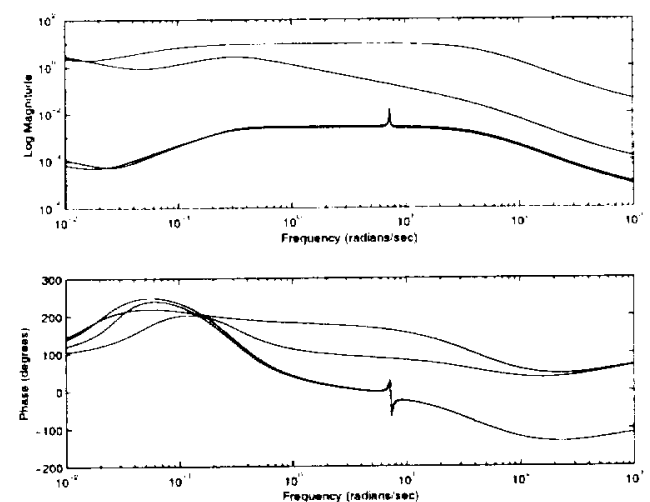

Fig. 5 Bode Plot for the $H_{\infty}$ Controller for Component No. 1

20 modes of the combined structure. The combined structure was also modeled globally (without synthesis) as a $20-\mathrm{m}$ long beam using its first 20 modes, resulting in a 40th-order state space model. This model was used for validation of the synthesis approach and is referred to as the truth model.

Table 2. Natural frequencies

\begin{tabular}{ccc} 
Mode No. & True & Synthesized \\
\hline 1 & 0 & 0 \\
2 & 0 & 0 \\
3 & 0.0410 & 0.0414 \\
4 & 0.1131 & 0.1158 \\
5 & 0.2217 & 0.2264 \\
6 & 0.3665 & 0.3676 \\
7 & 0.5475 & 0.5500 \\
8 & 0.7647 & 0.7809 \\
9 & 1.0181 & 1.0389 \\
10 & 1.3077 & 1.3108 \\
11 & 1.6335 & 1.6437 \\
12 & 1.9955 & 2.0425 \\
13 & 2.3938 & 2.4466 \\
14 & 2.8282 & 2.8344 \\
15 & 3.2988 & 3.3251 \\
16 & 3.8056 & 3.9045 \\
17 & 4.3488 & 4.4533 \\
18 & 4.9278 & 4.9389 \\
19 & 5.5432 & 5.6002 \\
20 & 6.1948 & 6.3764 \\
\hline
\end{tabular}

Using the procedure outlined in Eqs. (11)-(13), the dynamics of the two components were synthesized (combined). Table 2 compares the natural frequencies of the synthesized model vs. that of 


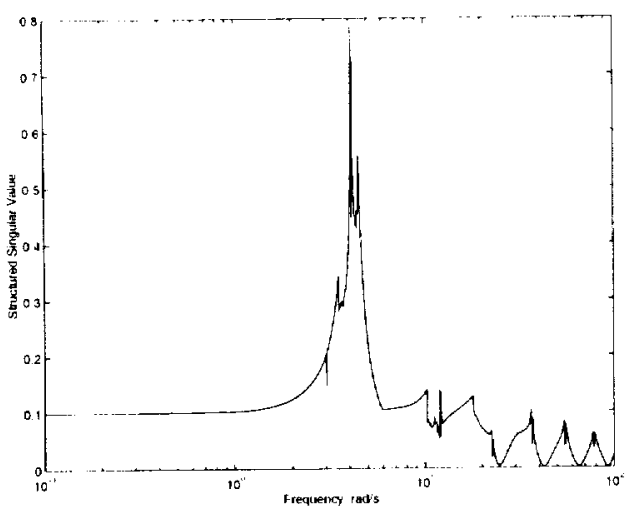

Fig. 6 Robust Stability $\mu$ Plot for the Synthesized System

the validation (truth) model. Comparison of the frequencies indicate a good match between the two models.

Now, both the synthesized model as well as the truth model were assessed against uncertainties in the frequencies and damping of the two components. Specifically, it was assumed that there is a five percent uncertainty associated with the frequency of the first mode in each component. Furthermore, it was assumed that there is a $\mathbf{1 5}$ percent uncertainty associated with the damping of the first mode in each component. The inherent structural damping ratio used for each of the component were chosen at 0.5 percent. The damping ratio used for the truth model was based on the values obtained from the synthesized model.

First, robust stability of the synthesized model was assessed. Following Eq. (23), the coefficients $Q_{i}$ for the four uncertainty terms in the state matrices of the components, corresponding to frequency and damping in their first mode, were defined and factored according to Eq. (24). Note that the uncertainties were assumed to be distinct, real scalars. Using the system defined in Eq. (21) (with appropriate input/output matrices for the uncertainties) together with the $H_{\infty}$ controller designed for component no. 1, a robust stability analysis was performed with aid of the "mu" routine of $\mu$-Analysis and Synthesis Toolbox. ${ }^{8}$ A Mu plot of the closed-loop system is provided in Fig. 6, where it is observed that the structured singular value $\mu$ is less than one for all frequencies. Therefore, the synthesized system with the $H_{\infty}$ controller is robustly stable.

Next, robust stability of the truth model against the same uncertainties in the component models was assessed. The first task was to approximately translate the uncertainties from the component level to the system level. This was performed by trans-

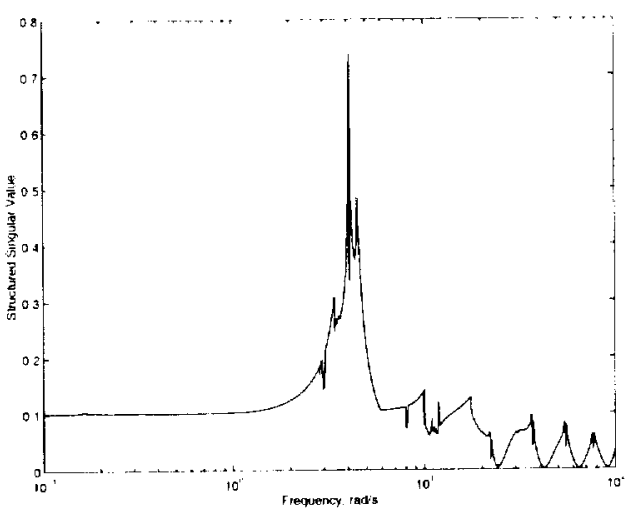

Fig. 7 Robust Stability $\mu$ Plot for the Truth System

forming the state matrix of the synthesized system to real modal form, with eigenvalues in increasing order on the diagonal. Then, Eq. (31) was used to determine the influence matrices at the system level, and the truth model given by Eq. (26) was used in robust stability analysis. Again, the $H_{\infty}$ controller designed for component no. 1 was the controller used in the robustness analysis of the closed-loop truth model. A Mu plot of the closed-loop system is provided in Fig. 7, where it is observed that the structured singular value $\mu$ is less than one for all frequencies. Comparing Figs. 6 and 7 , it is observed the $\mathrm{Mu}$ plots for the synthesized and truth systems match each other reasonably well. This validates the approximate characterization of the uncertainty at the system level from component level definitions.

\section{Concluding Remarks}

Robustness of flexible systems in the presence of model uncertainties at the component level was considered. Specifically, an approach for formulating robustness of flexible systems in the presence of frequency and damping uncertainties at the component level was presented. A controls-based algorithm for component mode synthesis was followed and modified to avoid to superfluous modes that may come about in the dynamics. First, robustness of synthesized systems was formulated in a Linear Fractional Transformation framework. The formulation was then extended to deal with global (non-synthesized) dynamic models with component-level uncertainties by projecting uncertainties from component levels to system level. A numerical example involving a twodimensional simulated docking problem was worked out to demonstrate the feasibility of the proposed approach. The results demonstrated the effectiveness of the robustness formulation in transformed coordinates and the efficacy of the projection approach to translate uncertainties from their component-level 
structures to system-level structures.

\section{References}

${ }^{1}$ Hurty, W., "Vibrations of Structural systems by Component Mode Syuthesis," Journal of Engineering Mechanics Division, Proc. of ASCE, 1960, pp. 51-69.

${ }^{2}$ Craig, R.R., J. and Bampton, M., "Coupling of Substructures for Dynamic Analysis," AIAA Journal, Vol. 6, No. 7, 1968, pp. 1313-9.

${ }^{3}$ Hintz, R., "Analytical Methods in Component Mode Synthesis," AlAA Journal, Vol. 13, No. 8, 1975, pp. 100716.

${ }^{4}$ Craig, R.R., J., "Methods of Component Mode Synthesis," Shock and Vibration Digest, Vol. 9, No. 11, 1977, pp. 310.

${ }^{5}$ Craig, R.R., J., Structural Dynamics: Introduction to Computer Methods, Wiley, 1981.

${ }^{6}$ Meirovitch, L. and Hale, $A$., "On the Substructure Synthesis Method," AIAA Journal, Vol. 19, No. 7, 1981, pp. $940-47$.

${ }^{7}$ Maghami, P. G. and Lim, K. B., "Substructure Synthesis: A Controls Approach," Proc. of Guidance, Navigation, and Controls Conf., Portland, OR, 1999.

${ }^{8}$ Balas, G. J., Doyle, J. C., Glover, K., Packard, A., and Smith, R., $\ell$-Analysis and Synthesis Toolbox, The MathWorks, Inc., 1995. 